\title{
A CENTRAL LIMIT THEOREM, AND RELATED RESULTS, FOR A TWO-COLOR RANDOMLY REINFORCED URN
}

\author{
GIACOMO ALETTI,* Università degli Studi di Milano \\ CATERINA MAY, ${ }^{* *}$ Università del Piemonte Orientale \\ PIERCESARE SECCHI, ${ }^{* * *}$ Politecnico di Milano
}

\begin{abstract}
We prove a central limit theorem for the sequence of random compositions of a two-color randomly reinforced urn. As a consequence, we are able to show that the distribution of the urn limit composition has no point masses.
\end{abstract}

Keywords: Reinforced processes; generalized Pólya urn; convergence of conditional distributions

2000 Mathematics Subject Classification: Primary 60F05

\section{Introduction}

Consider an urn initially containing $x$ balls of color black and $y$ balls of color white, with $x$ and $y$ nonnegative real numbers such that $x+y>0$. The urn is sequentially sampled: whenever the color of the sampled ball is black, the ball is replaced in the urn together with a random number of black balls, generated at that instant from a distribution $\mu$ with nonnegative bounded support; whenever the sampled ball is white, the ball is replaced in the urn together with a random number of balls, generated at that instant from a distribution $v$ with nonnegative bounded support. This is an informal description of the randomly reinforced urn (RRU) introduced in [13] and studied in [1], [2], [4], [7]-[10] under various assumptions concerning the reinforcement distributions $\mu$ and $v$. The urn has an interesting potential for applications since it describes a general model for reinforcement learning (see [2] and [7]); in clinical trials, it implements an optimal response adaptive design (see [5], [9], [11], and [14]).

The focus of this paper is on the asymptotic behavior of the sequence $\left\{Z_{n}\right\}$ describing the random proportions of black balls in the urn along the sampling sequence; in [13] it was proved that the sequence $\left\{Z_{n}\right\}$ converges almost surely to a random limit $Z_{\infty} \in[0,1]$.

When $\mu=v$, an RRU is a special case of the generalized Pólya urn studied by Crimaldi in [3]; for the sequence of random proportions $\left\{Z_{n}\right\}$ generated by her urn, Crimaldi proved a central limit theorem by showing almost-sure conditional convergence to a Gaussian kernel of the sequence $\left\{\sqrt{n}\left(Z_{n}-Z_{\infty}\right)\right\}$. Crimaldi's result does not hold for a general RRU; in this paper we extend it to cover the case of an RRU with reinforcement distributions $\mu$ and $v$ having the same mean. When the means of $\mu$ and $v$ are different, the limit proportion $Z_{\infty}$ of an RRU is

\footnotetext{
Received 20 January 2009; revision received 5 June 2009.

* Postal address: Dipartimento di Matematica 'F. Enrigues', Università degli Studi di Milano, Via Cesare Saldini 50, 20133 Milano, Italy. Email address: giacomo.aletti@mat.unimi.it

** Postal address: Dipartimento SEMEQ, Università del Piemonte Orientale, via Perrone 18, 28100 Novara, Italy.

Email address: caterina.may@eco.unipmn.it

*** Postal address: MOX - Modellistica e Calcolo Scientifico, Dipartimento di Matematica 'F. Brioschi', Politecnico di Milano, via Bonardi 9, 20133 Milano, Italy. Email address: piercesare.secchi@polimi.it
} 
a point mass either in 1 or in 0 , according to the reinforcement distribution having the larger mean, as proved with different arguments in [2], [7], and [13].

A nice implication of our RRU central limit theorem is that we are now able to prove that the distribution of the limit proportion $Z_{\infty}$ has no point masses in $[0,1]$, when the means of the reinforcement distributions are the same. This gives a new drive to the problem concerning the absolute continuity of the distribution of the limit proportion of a generalized Pólya urn, considered, for instance, in [15].

The paper is organized as follows. In the next section we will formally introduce the RRU model along with the notation used in the paper. The main results of the paper are stated in Section 3, and the proofs appear in Section 4. A remark on the absolute continuity of the distribution of $Z_{\infty}$ concludes the paper.

\section{Model description and notation}

On a rich enough probability space $(\Omega, \mathcal{A}, \mathrm{P})$, define two independent infinite sequences of random elements, $\left\{U_{n}\right\}$ and $\left\{\left(V_{n}, W_{n}\right)\right\} ;\left\{U_{n}\right\}$ is a sequence of independent and identically distributed (i.i.d.) random variables uniformly distributed on $[0,1]$, while $\left\{\left(V_{n}, W_{n}\right)\right\}$ is a sequence of i.i.d. bivariate random vectors with components uniformly distributed on $[0,1]$. Given two probability distributions $\mu$ and $v$ on $[0, \beta]$, with $\beta>0$, indicate their quantile functions with $q_{\mu}$ and $q_{\nu}$, respectively. Then, define an infinite sequence $\left\{\left(R_{X}(n), R_{Y}(n)\right)\right\}$ of bivariate random vectors by setting, for all $n$,

$$
R_{X}(n)=q_{\mu}\left(V_{n}\right) \quad \text { and } \quad R_{Y}(n)=q_{v}\left(W_{n}\right) .
$$

Note that, whereas the sequences $\left\{\left(R_{X}(n), R_{Y}(n)\right)\right\}$ and $\left\{U_{n}\right\}$ are independent, the random variables $R_{X}(n)$ and $R_{Y}(n)$ might be dependent; however, for every $n$, their distributions are $\mu$ and $\nu$, respectively. We indicate with $m_{\mu}$ and $m_{v}$, and with $\sigma_{\mu}^{2}$ and $\sigma_{v}^{2}$ the means and the variances of two random variables $R_{X}$ and $R_{Y}$ having probability distributions $\mu$ and $\nu$, respectively.

We are now ready to introduce a process whose law is that of an RRU, as defined in [13]. Let $x$ and $y$ be two nonnegative real numbers such that $x+y>0$. Set $X_{0}=x$ and $Y_{0}=y$, and, for $n=0,1,2, \ldots$, let

$$
X_{n+1}=X_{n}+R_{X}(n+1) \delta_{n+1}, \quad Y_{n+1}=Y_{n}+R_{Y}(n+1)\left(1-\delta_{n+1}\right),
$$

where the variable $\delta_{n+1}$ is the indicator of the event $\left\{U_{n+1} \leq X_{n}\left(X_{n}+Y_{n}\right)^{-1}\right\}$. The law of $\left\{\left(X_{n}, Y_{n}\right)\right\}$ is that of the stochastic process counting, along the sampling sequence, the number of black and white balls present in an RRU with initial composition $(x, y)$ and reinforcement distributions equal to $\mu$ and $\nu$, respectively.

For $n=0,1,2, \ldots$, let

$$
Z_{n}=\frac{X_{n}}{X_{n}+Y_{n}}
$$

$Z_{n}$ represents the proportion of black balls in the urn before the $(n+1)$ th ball is sampled from it. In [13] it was proved that $\left\{Z_{n}\right\}$ is eventually a bounded submartingale or supermartingale, according to the mean of $\mu$ being larger or smaller than that of $\nu$. Hence, for $n$ growing to $\infty$, $Z_{n}$ converges almost surely, and in $L^{p}, 1 \leq p \leq \infty$, to a random variable $Z_{\infty} \in[0,1]$. 
For $n=1,2, \ldots$, let $R_{n}=\delta_{n} R_{X}(n)+\left(1-\delta_{n}\right) R_{Y}(n)$ be the urn reinforcement, when the urn is sampled for the $n$th time, and set

$$
\begin{gathered}
Q_{n-1}^{X}=\frac{R_{X}(n)}{\sum_{i=1}^{n} R_{i}}, \quad Q_{n-1}^{Y}=\frac{R_{Y}(n)}{\sum_{i=1}^{n} R_{i}}, \\
Q_{n-1}=\frac{R_{n}}{\sum_{i=1}^{n} R_{i}}=\delta_{n} Q_{n-1}^{X}+\left(1-\delta_{n}\right) Q_{n-1}^{Y},
\end{gathered}
$$

with $Q_{n-1}^{X}=Q_{n-1}^{Y}=Q_{n-1}=1$ if $R_{i}=0$ for all $i=1, \ldots, n$. For shortness, we will write $D_{n}$ for the random number $X_{n}+Y_{n}$, interpreted as the size of the urn before it is sampled for the $(n+1)$ th time. Clearly, $D_{0}=x+y$ while $D_{n+1}=D_{n}+R_{n+1}$ for $n=0,1,2, \ldots$ Finally, let $\mathcal{A}_{n}=\sigma\left(U_{1}, \ldots, U_{n},\left(V_{1}, W_{1}\right), \ldots,\left(V_{n}, W_{n}\right)\right)$ and consider the filtration $\left\{\mathcal{A}_{n}\right\}$; for $n=1,2, \ldots$, we indicate with $M_{n}$ and $A_{n}$ the two terms given by Doob's semimartingale decomposition of $Z_{n}$, i.e.

$$
Z_{n}=Z_{0}+M_{n}+A_{n}
$$

where $\left\{M_{n}\right\}$ is a zero-mean martingale with respect to $\left\{\mathcal{A}_{n}\right\}$, while $\left\{A_{n}\right\}$ is previsible with respect to $\left\{\mathcal{A}_{n}\right\}$. Theorem 2 of [13] shows that $\left\{A_{n}\right\}$ is eventually increasing or decreasing.

\section{Main results}

For every set $A \in \mathcal{A}$, every $\omega \in \Omega$, and $n=1,2, \ldots$, define

$$
K_{n}(\omega, A)=\mathrm{P}\left(\sqrt{n}\left(Z_{n}-Z_{\infty}\right) \in A \mid \mathcal{A}_{n}\right)(\omega),
$$

i.e. $K_{n}$ is a version of the conditional distribution of $\sqrt{n}\left(Z_{n}-Z_{\infty}\right)$ given $\mathcal{A}_{n}$. When the reinforcement distributions of an RRU are the same, i.e. $\mu=v$, and $\mu$ is different from the point mass at 0 , Corollary 4.1 of [3] shows that, for almost every $\omega \in \Omega$, the sequence of probability distributions $\left\{K_{n}(\omega, \cdot)\right\}$ converges weakly to the Gaussian distribution

$$
N\left(0, h Z_{\infty}(\omega)\left(1-Z_{\infty}(\omega)\right)\right)
$$

where

$$
h=\frac{\int_{0}^{\beta} k^{2} \mu(\mathrm{d} k)}{\left(\int_{0}^{\beta} k \mu(\mathrm{d} k)\right)^{2}} .
$$

The next theorem extends this central limit result to a general RRU with reinforcement distributions $\mu$ and $v$ having the same mean.

Theorem 1. Assume that $m_{\mu}=m_{v}=m>0$. Let

$$
H=m^{-2}\left(Z_{\infty} \int_{0}^{\beta} k^{2} v(\mathrm{~d} k)+\left(1-Z_{\infty}\right) \int_{0}^{\beta} k^{2} \mu(\mathrm{d} k)\right) .
$$

Then, for almost every $\omega \in \Omega$, the sequence of probability distributions $\left\{K_{n}(\omega, \cdot)\right\}$ converges weakly to the Gaussian distribution

$$
N\left(0, H(\omega) Z_{\infty}(\omega)\left(1-Z_{\infty}(\omega)\right)\right)
$$


Note that, in Theorem 1, provided that the initial urn composition $(x, y)$ is such that $\min (x, y)>0$, the variance $H(\omega) Z_{\infty}(\omega)\left(1-Z_{\infty}(\omega)\right)$ is strictly positive for almost every $\omega \in \Omega$, as guaranteed by Theorem 3.2 of [9]; hence, the limit distribution of $K_{n}$ is absolutely continuous. Indeed, May and Flournoy [9] proved that equality of the means of the reinforcement distributions implies that $\mathrm{P}\left(Z_{\infty}=0\right)=\mathrm{P}\left(Z_{\infty}=1\right)=0$. In the particular case when $\mu=v$, the distribution of $Z_{\infty}$ has no point masses at all; this has been shown in [10]. As a consequence of Theorem 1, we are now able to prove that this is also true with the sole assumption that the means of $\mu$ and $v$ are the same.

Theorem 2. If $\min (x, y)>0$ and $m_{\mu}=m_{v}=m>0$, the distribution of $Z_{\infty}$ has no point masses. That is, $\mathrm{P}\left(Z_{\infty}=p\right)=0$ for all $p \in[0,1]$.

When $m_{\mu} \neq m_{\nu}$, the distribution of $Z_{\infty}$ is the point mass at 1 or at 0 , according to whether $m_{\mu}$ is larger or smaller than $m_{\nu}$; this has been proved in [2], [7], and [13] under the assumption that the supports of $\mu$ and $v$ are bounded away from 0 . Within the framework of the present paper, we are able to show that the result holds more generally when the supports of $\mu$ and $\nu$ are contained in the interval $[0, \beta]$.

Theorem 3. Assume that $m_{\mu}>m_{v}$. Then $\mathrm{P}\left(Z_{\infty}=1\right)=1$.

\section{Proofs and auxiliary results}

The proof of Theorem 1 will make use of a few auxiliary results, which we state and prove as Lemmas $1-7$, below.

From now on, given a sequence $\left\{\xi_{n}\right\}$ of random variables, we will denote by $\Delta \xi_{n+1}$ the increments $\left(\xi_{n+1}-\xi_{n}\right)$. Moreover, given any two sequences $\left\{a_{n}\right\}$ and $\left\{b_{n}\right\}$ of real numbers, we will use the symbol $a_{n} \backsim b_{n}$ to denote that $a_{n} / b_{n} \rightarrow 1$ as $n \rightarrow \infty$.

Lemma 1. Let $R$ and $D$ be two random variables defined on $(\Omega, \mathcal{A}, \mathrm{P})$ with values in $B_{R}=$ $[0, \beta]$ and $B_{D}=[0, \infty)$, respectively, and let $g$ be a sub-sigma-field of $\mathcal{A}$ such that $R$ is independent of $g$ while $D$ is measurable with respect to $g$. Let $h$ be a measurable real-valued function defined on $B_{R} \times B_{D}$ and such that $h(\cdot, t)$ is convex for all $t \in B_{D}$. Then, for almost every $\omega \in \Omega$,

$$
h(\mathrm{E}(R), D(\omega)) \leq \mathrm{E}(h(R, D) \mid \mathcal{g})(\omega) \leq\left(\frac{\mathrm{E}(R)}{\beta} h(\beta, D(\omega))+\frac{\beta-\mathrm{E}(R)}{\beta} h(0, D(\omega))\right) .
$$

The above inequalities are reversed if $h(\cdot, t)$ is concave for all $t \in B_{D}$.

Proof. If $\pi$ is the probability distribution of $R$,

$$
\mathrm{E}(h(R, D) \mid \mathcal{g})(\omega)=\int_{0}^{\beta} h(x, D(\omega)) \pi(\mathrm{d} x)
$$

for almost every $\omega \in \Omega$. The left-hand inequality is now an instance of Jensen's inequality. The right-hand inequality follows after noticing that

$$
h(x, t) \leq x \frac{h(\beta, t)}{\beta}+\frac{\beta-x}{\beta} h(0, t)
$$

for all $(x, t) \in B_{R} \times B_{D}$, since $h(\cdot, t)$ is convex. 
As a consequence of the previous lemma, we can bound the increments $\Delta A_{n}$ of the compensator process $\left\{A_{n}\right\}$. First note that, for all $n=0,1,2, \ldots$,

$$
\Delta A_{n+1}=\mathrm{E}\left(\Delta Z_{n+1} \mid \mathcal{A}_{n}\right)=Z_{n}\left(1-Z_{n}\right) A_{n+1}^{*}
$$

almost surely, where

$$
A_{n+1}^{*}=\mathrm{E}\left(\frac{R_{X}(n+1) / D_{n}}{1+R_{X}(n+1) / D_{n}}-\frac{R_{Y}(n+1) / D_{n}}{1+R_{Y}(n+1) / D_{n}} \mid \mathcal{A}_{n}\right) .
$$

Lemma 2. If $m_{\mu}>m_{v}$, for almost every $\omega \in \Omega$, there is an $a>0$ such that

$$
A_{n+1}^{*}(\omega) \geq \frac{a}{D_{n}(\omega)}
$$

eventually. If $m_{\mu}=m_{v}=m>0$, for $n=0,1,2, \ldots$ and almost every $\omega \in \Omega$,

$$
\left|A_{n+1}^{*}(\omega)\right| \leq \frac{m(\beta-m)}{\left(\beta+D_{n}(\omega)\right)\left(m+D_{n}(\omega)\right)}=O\left(\frac{1}{D_{n}^{2}(\omega)}\right) .
$$

Proof. Note that $h(x, t)=x /(x+t)$ is a concave bounded function of $x \geq 0$ for any fixed $t \geq 0$. For $n=0,1,2, \ldots$,

$$
A_{n+1}^{*}=\mathrm{E}\left(h\left(R_{X}(n+1), D_{n}\right)-h\left(R_{Y}(n+1), D_{n}\right) \mid \mathcal{A}_{n}\right)
$$

hence, by applying Lemma 1 we obtain

$$
A_{n+1}^{*} \geq \frac{m_{\mu}}{\beta+D_{n}}-\frac{m_{v}}{m_{v}+D_{n}}=\frac{D_{n}\left(m_{\mu}-m_{v}\right)-m_{v}\left(\beta-m_{\mu}\right)}{\left(m_{v}+D_{n}\right)\left(\beta+D_{n}\right)}
$$

and

$$
A_{n+1}^{*} \leq \frac{m_{\mu}}{m_{\mu}+D_{n}}-\frac{m_{v}}{\beta+D_{n}}=\frac{D_{n}\left(m_{\mu}-m_{v}\right)+m_{\mu}\left(\beta-m_{v}\right)}{\left(m_{\mu}+D_{n}\right)\left(\beta+D_{n}\right)}
$$

on a set of probability 1 . The thesis is now a consequence of the fact that $\lim _{n \rightarrow \infty} D_{n}=\infty$ almost surely (see, e.g. [9, Proposition 2.3, Proposition 2.4]).

Indeed, when $m_{\mu}=m_{v}=m>0$, two educational cases emerge by inspection of $A_{n+1}^{*}$ in the light of Lemma 1. The first case is when $\mu$ is the point mass at $m$; then $A_{n+1}^{*} \geq 0$ for all $n=0,1,2, \ldots$ and the process $\left\{Z_{n}\right\}$ is a bounded submartingale. At the other extreme, let $\mu$ be the distribution of the random variable $\beta \zeta$, with $\zeta$ distributed according to a Bernoulli $(m / \beta)$; then $A_{n+1}^{*} \leq 0$ for all $n=0,1,2, \ldots$ and the process $\left\{Z_{n}\right\}$ is a bounded supermartingale.

In [9, Lemma A.1(iii)] it was proved that $\lim _{n \rightarrow \infty} D_{n} / n=m$ almost surely when $m_{\mu}=$ $m_{v}=m>0$. The next lemma improves our general understanding of the growth speed of the urn size $D_{n}$.

Lemma 3. Assume that $\min \left(m_{\mu}, m_{v}\right)>0$. For all $c, \alpha \geq 0$, there are two constants $0<a_{1}<$ $a_{2}<\infty$ such that

$$
\frac{a_{1}}{n^{\alpha}} \leq \mathrm{E}\left(\frac{1}{\left(c+D_{n}\right)^{\alpha}}\right) \leq \frac{a_{2}}{n^{\alpha}}
$$

eventually. Moreover, if $m_{\mu}=m_{v}=m>0$ then

$$
\mathrm{E}\left(\frac{1}{\left(c+D_{n}\right)^{\alpha}}\right) \sim \frac{1}{\left(c+D_{0}+m n\right)^{\alpha}} .
$$


Proof. It is trivial to prove the lemma when the supports of $\mu$ and $v$ are both bounded away from 0 ; this is the case, for instance, when $\mu$ and $v$ are both point masses at real numbers different from 0 . For the general case, set $\sigma^{2}=\min \left(\sigma_{\mu}^{2}, \sigma_{v}^{2}\right)$ and assume that $\max \left(\sigma_{\mu}^{2}, \sigma_{v}^{2}\right)>0$; without loss of generality, we also assume that $m_{\mu} \geq m_{v}>0$.

The left-hand inequality in (3) follows from Jensen's inequality:

$$
\mathrm{E}\left(\frac{1}{\left(c+D_{n}\right)^{\alpha}}\right) \geq \frac{1}{\left(c+\mathrm{E}\left(D_{n}\right)\right)^{\alpha}} \geq \frac{1}{\left(c+D_{0}+n m_{\mu}\right)^{\alpha}} .
$$

To prove the right-hand inequality, we consider the following two cases.

Case 1: $\sigma>0$. For $n=1,2, \ldots$ and $i=1, \ldots, n$, set

$$
L_{n i}=\frac{\delta_{i}\left(R_{X}(i)-m_{\mu}\right)+\left(1-\delta_{i}\right)\left(R_{Y}(i)-m_{v}\right)}{\sqrt{n} \sqrt{Z_{i-1} \sigma_{\mu}^{2}+\left(1-Z_{i-1}\right) \sigma_{v}^{2}}} \leq \frac{R_{i}-m_{v}}{\sqrt{n} \sigma} .
$$

Then we can show that $\left\{L_{n i}, \widetilde{F}_{n i}=\mathcal{A}_{i}, n=1,2, \ldots, i=1, \ldots, n\right\}$ is a martingale difference array such that, for all $n=1,2, \ldots$, and $i=1, \ldots, n$,

$$
\left|L_{n i}\right| \leq \frac{\beta}{\sigma \sqrt{n}}
$$

while

$$
\sum_{i=1}^{n} \mathrm{E}\left(L_{n i}^{2} \mid \mathcal{F}_{n i-1}\right)=1
$$

with probability 1 .

Let $S_{n}=\sum_{i=1}^{n} L_{n i}$. Then, by the large deviations result [18, Theorem 1] for martingales and (4), we obtain

$$
\limsup _{n \rightarrow \infty} \frac{\mathrm{P}\left(\sum_{i=1}^{n} R_{i} \leq n m_{v}-\sigma n^{5 / 8}\right)}{\Phi\left(-n^{1 / 8}\right)} \leq \lim _{n \rightarrow \infty} \frac{\mathrm{P}\left(S_{n} \leq-n^{1 / 8}\right)}{\Phi\left(-n^{1 / 8}\right)}=1,
$$

where $\Phi$ indicates the standard normal distribution. Since

$$
\Phi(-x) \leq \frac{\exp \left(-x^{2} / 2\right)}{x \sqrt{2 \pi}} \quad \text { for all } x>0
$$

we obtain

$$
\mathrm{P}\left(\sum_{i=1}^{n} R_{i} \leq n m_{v}-\sigma n^{5 / 8}\right) \leq \frac{\exp \left(-n^{1 / 4} / 2\right)}{n^{1 / 8}} \text { eventually. }
$$

For $n=1,2, \ldots$, set $F_{n}=\left\{\sum_{i=1}^{n} R_{i} \leq n m_{v}-\sigma n^{5 / 8}\right\}$; then

$$
\begin{aligned}
\mathrm{E}\left(\frac{1}{\left(c+D_{n}\right)^{\alpha}}\right)= & \frac{1}{\left(c+D_{0}\right)^{\alpha}} \mathrm{E}\left(\frac{1}{\left(1+\sum_{i=1}^{n} R_{i} /\left(c+D_{0}\right)\right)^{\alpha}} ; F_{n}\right) \\
& +\mathrm{E}\left(\frac{1}{\left(c+D_{0}+\sum_{i=1}^{n} R_{i}\right)^{\alpha}} ; F_{n}^{c}\right) \\
\leq & \frac{\mathrm{P}\left(F_{n}\right)}{\left(c+D_{0}\right)^{\alpha}}+\frac{1}{\left(c+D_{0}+n m_{v}-\sigma n^{5 / 8}\right)^{\alpha}} \\
\leq & \frac{1}{\left(c+D_{0}+n m_{v}\right)^{\alpha}}\left(\left(\frac{c+D_{0}+m_{v}}{c+D_{0}}\right)^{\alpha} n^{\alpha} \mathrm{P}\left(F_{n}\right)+\frac{1}{(1+o(1))^{\alpha}}\right) .
\end{aligned}
$$


Hence, by (5),

$$
\limsup _{n \rightarrow \infty} \frac{\mathrm{E}\left(1 /\left(c+D_{n}\right)^{\alpha}\right)}{1 /\left(c+D_{0}+m_{Y} n\right)^{\alpha}} \leq 1
$$

and this completes the proof of this case.

Case 2: $\sigma=0$. Assume that $\sigma_{\mu}^{2}>0$ (the case $\sigma_{v}^{2}>0$ is analogous). Hence, $v$ is the point mass at $m_{v}>0$. Let $\left\{\widetilde{R}_{Y}(n)\right\}$ be a sequence of i.i.d. random variables, independent of $\left\{\mathcal{A}_{n}\right\}$ and such that each variable $\widetilde{R}_{Y}(n)$, when multiplied by $m_{\mu} / m_{\nu}$, has probability distribution equal to $\mu$. For $n=1,2, \ldots$, define

$$
\tau_{n}^{X}=\inf \left\{k: \sum_{i=1}^{k} \delta_{i} \geq n\right\}, \quad \tau_{n}^{Y}=\inf \left\{k: \sum_{i=1}^{k}\left(1-\delta_{i}\right) \geq n\right\} .
$$

By Jensen's inequality and [9, Proposition 2.4], we then have

$$
\begin{aligned}
\mathrm{E}\left(\frac{1}{\left(c+D_{n}\right)^{\alpha}}\right) & =\mathrm{E}\left(\mathrm{E}\left(\frac{1}{\left(c+D_{n}\right)^{\alpha}} \mid \sum_{i=1}^{n} \delta_{i}=k ; \mathcal{A}_{n}\right)\right) \\
& =\mathrm{E}\left(\mathrm{E}\left(\frac{1}{\left(c+D_{0}+\sum_{i=1}^{k} R_{X}\left(\tau_{i}\right)+(n-k) m_{\nu}\right)^{\alpha}} \mid \sum_{i=1}^{n} \delta_{i}=k ; \mathcal{A}_{n}\right)\right) \\
& \leq \mathrm{E}\left(\mathrm{E}\left(\frac{1}{\left(c+D_{0}+\sum_{i=1}^{k} R_{X}\left(\tau_{i}^{X}\right)+\sum_{i=1}^{n-k} \widetilde{R}_{Y}\left(\tau_{i}^{Y}\right)\right)^{\alpha}} \mid \sum_{i=1}^{n} \delta_{i}=k ; \mathcal{A}_{n}\right)\right) \\
& =\mathrm{E}\left(\frac{1}{\left(c+D_{0}+\sum_{i=1}^{n}\left(\delta_{i} R_{X}(i)+\left(1-\delta_{i}\right) \widetilde{R}_{Y}(i)\right)\right)^{\alpha}}\right) .
\end{aligned}
$$

Since $\min \left(\sigma_{\mu}^{2}, \operatorname{var}\left(\widetilde{R}_{Y}\right)\right)=\left(m_{v} / m_{\mu}\right)^{2} \sigma_{\mu}^{2}>0$, case 1 applied to a coupled RRU with the same initial composition and reinforcements equal to $R_{X}(n)$ whenever $\delta(n)=1$, and $\widetilde{R}_{Y}(n)$ whenever $\delta_{n}=0$, yields the thesis.

Lemma 4. Assume that $m_{\mu}=m_{v}=m>0$. Then

$$
\mathrm{E}\left(\sum_{k>0} \sqrt{k}\left|\Delta A_{k}\right|\right)<\infty
$$

Proof. Lemma 3 and Lemma 2 yield

$$
\sum_{k>0} \sqrt{k} \mathrm{E}\left(\left|\Delta A_{k}\right|\right) \leq K_{1} \sum_{k>0} \sqrt{k} \mathrm{E}\left(\frac{1}{\left(m+D_{k}\right)^{2}}\right) \leq K_{2} \sum_{k>0} k^{-3 / 2}<\infty
$$

for suitable constants $K_{1}, K_{2}>0$.

Lemma 5. We have

$$
\mathrm{E}\left(\sum_{k=0}^{\infty} k^{2} Q_{k}^{4}\right)<\infty, \quad \mathrm{E}\left(\sum_{k=0}^{\infty} k^{2}\left(Q_{k}^{X}\right)^{4}\right)<\infty, \quad \mathrm{E}\left(\sum_{k=0}^{\infty} k^{2}\left(Q_{k}^{Y}\right)^{4}\right)<\infty .
$$

Proof. For all $x>0$ and $0 \leq a \leq b$,

$$
\left(\frac{a}{b}\right)^{4} \leq\left(\frac{a+x}{b+x}\right)^{4}
$$


if $0 / 0$ is set equal to 1 . Then, for $k=0,1, \ldots$,

$$
Q_{k}^{4}=\left(\frac{R_{k+1}}{\sum_{i=1}^{k+1} R_{i}}\right)^{4} \leq\left(\frac{R_{k+1}+1+D_{0}}{1+D_{k+1}}\right)^{4} \leq\left(1+D_{0}+\beta\right)^{4}\left(\frac{1}{1+D_{k}}\right)^{4} .
$$

It follows from Lemma 3 with $\alpha=4$ that

$$
\sum_{k=0}^{\infty} k^{2} \mathrm{E}\left(Q_{k}^{4}\right) \leq K \sum_{k>0} k^{-2}<\infty
$$

for a suitable constant $K>0$. Hence, $\mathrm{E}\left(\sum_{k>0} k^{2} Q_{k}^{4}\right)<\infty$. The proof is similar for $Q^{X}$ and $Q^{Y}$ : replace $R_{k+1}$ with $R_{X}(k+1)$ or $R_{Y}(k+1)$, respectively, in the numerator of the first two terms of (6).

The next lemma is an auxiliary result which will be used for proving the almost-sure convergence of random series.

Lemma 6. Let $\left\{a_{k}\right\},\left\{b_{k}\right\}$, and $\left\{c_{k}\right\}$ be three infinite sequences of real, nonnegative numbers such that $b_{k}$ and $c_{k}$ are eventually strictly positive, $b_{k} \sim c_{k}$, and $\sum_{k} a_{k} / b_{k}<\infty$. Then

$$
\sum_{k>n} \frac{a_{k}}{b_{k}} \sim \sum_{k>n} \frac{a_{k}}{c_{k}} \text { as } n \rightarrow \infty
$$

Proof. For lack of a reference, we prove the lemma. For a fixed $0<\varepsilon \leq \frac{1}{2}$, let $n_{0}$ be large enough that $b_{k}$ and $c_{k}$ are strictly positive and $(1-\varepsilon) b_{k} \leq c_{k} \leq(1+\varepsilon) b_{k}$ for $k>n_{0}$. Then, for $n \geq n_{0}$,

$$
(1-2 \varepsilon) \sum_{k>n} \frac{a_{k}}{b_{k}} \leq \sum_{k>n} \frac{a_{k}}{(1+\varepsilon) b_{k}} \leq \sum_{k>n} \frac{a_{k}}{c_{k}} \leq \sum_{k>n} \frac{a_{k}}{(1-\varepsilon) b_{k}} \leq(1+2 \varepsilon) \sum_{k>n} \frac{a_{k}}{b_{k}} .
$$

Finally, we need a general fact about the convergence of random sequences; for lack of a better reference, see [16, Lemma 3.2].

Lemma 7. Let $\left\{\xi_{n}\right\}$ be a sequence of real random variables adapted to the filtration $\left\{\mathcal{A}_{n}\right\}$. If $\mathrm{P}\left(\xi_{1}<\infty\right)=1$ and

$$
\sum_{n} \mathrm{E}\left(\xi_{n+1} \mid \mathcal{A}_{n}\right)<\infty \text { and } \sum_{n} \mathrm{E}\left(\xi_{n+1}^{2} \mid \mathcal{A}_{n}\right)<\infty
$$

almost surely, then $\sum_{n} \xi_{n}$ converges almost surely.

We can now demonstrate a proposition that will act as a cornerstone for the proof of the main result of the paper.

Proposition 1. Assume that $m_{\mu}=m_{v}=m>0$, and let

$$
H_{X}=m^{-2} \mathrm{E}\left(R_{X}^{2}\right), \quad H_{Y}=m^{-2} \mathrm{E}\left(R_{Y}^{2}\right) .
$$

Then

$$
\lim _{n \rightarrow \infty} n \sum_{k>n}\left(Q_{k}^{X}\right)^{2}=H_{X}, \quad \lim _{n \rightarrow \infty} n \sum_{k>n}\left(Q_{k}^{Y}\right)^{2}=H_{Y}
$$

on a set of probability 1. 
Proof. We prove that $\lim _{n \rightarrow \infty} n \sum_{k>n}\left(Q_{k}^{X}\right)^{2}=H_{X}$ almost surely by an argument similar to that used to prove Corollary 4.1 of [3]. The proof that $\lim _{n \rightarrow \infty} n \sum_{k>n}\left(Q_{k}^{Y}\right)^{2}=H_{Y}$ almost surely is similar and will be omitted. Let $\rho=\mathrm{E}\left(R_{X}^{2}\right)$. The series

$$
\sum_{n} n^{-1}\left(R_{X}^{2}(n+1)-\rho\right)
$$

converges almost surely, since it is a series of zero-mean independent random variables with variances bounded by $n^{-2} \beta^{4}$. This fact and Abel's theorem imply that

$$
\lim _{n \rightarrow \infty} n \sum_{k>n} k^{-2}\left(R_{X}^{2}(k+1)-\rho\right)=0
$$

on a set of probability 1 . Then

$$
\lim _{n \rightarrow \infty} n \sum_{k>n} k^{-2} R_{X}^{2}(k+1)=\rho
$$

on a set of probability 1 , since $\lim _{n \rightarrow \infty} n \sum_{k>n} k^{-2}=1$.

From [9, Lemma A.1(iii)], it follows that $\lim _{k \rightarrow \infty}(m k)^{-1} \sum_{i=1}^{k} R_{i}=1$ almost surely and, thus,

$$
\left(Q_{k}^{X}\right)^{2} \sim m^{-2} k^{-2} R_{X}^{2}(k+1)
$$

on a set of probability 1 . Therefore, Lemma 6 implies that

$$
n \sum_{k \geq n}\left(Q_{k}^{X}\right)^{2} \sim m^{-2} n \sum_{k \geq n} k^{-2} R_{X}^{2}(k+1)
$$

almost surely; however, (7) shows that the right-hand term converges almost surely to $m^{-2} \rho=$ $H_{X}$ as $n \rightarrow \infty$. This concludes the proof of the proposition.

Proof of Theorem 1. For $n=0,1,2, \ldots$, set

$$
G_{n}=\sum_{k>n} \sqrt{k}\left|\Delta A_{k}\right| \geq 0 \quad \text { and } \quad W_{n}=\mathrm{E}\left(G_{n} \mid \mathcal{A}_{n}\right)
$$

Because of Lemma 4 , the process $\left\{G_{n}\right\}$ converges monotonically to 0 almost surely and in $L^{1}$, as $n$ goes to $\infty$. Hence, the process $\left\{W_{n}\right\}$, being a nonnegative supermartingale, converges to 0 almost surely and in $L^{1}$, as $n$ goes to $\infty$. Since, for $n=1,2, \ldots$,

$$
\mathrm{E}\left(\sqrt{n}\left|A_{\infty}-A_{n}\right| \mid \mathcal{A}_{n}\right) \leq \mathrm{E}\left(\sum_{k>n} \sqrt{k}\left|\Delta A_{k}\right| \mid \mathcal{A}_{n}\right)=\mathrm{E}\left(G_{n} \mid \mathcal{A}_{n}\right)
$$

almost surely, we obtain, for all $t>0$,

$$
\mathrm{P}\left(\sqrt{n}\left|A_{\infty}-A_{n}\right|>t \mid \mathcal{A}_{n}\right) \leq \frac{\mathrm{E}\left(\sqrt{n}\left|A_{\infty}-A_{n}\right| \mid \mathcal{A}_{n}\right)}{t} \leq \frac{\mathrm{E}\left(G_{n} \mid \mathcal{A}_{n}\right)}{t}
$$

on a set of probability 1 ; therefore, $\mathcal{L}\left(\sqrt{n}\left|A_{\infty}-A_{n}\right| \mid \mathcal{A}_{n}\right)(\omega)$ weakly converges to the mass function at 0 for almost every $\omega \in \Omega$. Proving the theorem is thus equivalent to showing that, 
for almost every $\omega \in \Omega, \mathcal{L}\left(\sqrt{n}\left(M_{n}-M_{\infty}\right) \mid \mathcal{A}_{n}\right)(\omega)$ weakly converges to a $\mathcal{N}(0, \bar{H}(\omega))$, where

$$
\bar{H}(\omega)=H(\omega) Z_{\infty}(\omega)\left(1-Z_{\infty}(\omega)\right) .
$$

Since $\left\{M_{n}\right\}$ is a martingale, this follows from [3, Proposition 2.2] once we show that

$$
\mathrm{E}\left(\sup _{k} \sqrt{k}\left|\Delta M_{k}\right|\right)<\infty
$$

and

$$
\lim _{n \rightarrow \infty} n \sum_{k>n}\left(\Delta M_{k}\right)^{2}=\bar{H} \quad \text { almost surely. }
$$

Proof of $(8 a)$. Since

$$
\sqrt{k}\left|\Delta M_{k}\right| \leq \sqrt{k}\left|\Delta A_{k}\right|+\sqrt{k}\left|\Delta Z_{k}\right|
$$

and

$$
\mathrm{E}\left(\sup _{k} \sqrt{k}\left|\Delta A_{k}\right|\right) \leq \sum_{k} \sqrt{k} \mathrm{E}\left(\left|\Delta A_{k}\right|\right)
$$

from Lemma 4 we obtain

$$
\mathrm{E}\left(\sup _{k} \sqrt{k}\left|\Delta M_{k}\right|\right)<\infty \quad \Longleftrightarrow \quad \mathrm{E}\left(\sup _{k} \sqrt{k}\left|\Delta Z_{k}\right|\right)<\infty .
$$

Note that, for $n=0,1,2, \ldots, \delta_{n+1} R_{X}(n+1)=\delta_{n+1} R_{n+1}$ and

$$
\begin{aligned}
Z_{n}-Z_{n+1} & =\frac{X_{n}}{D_{n}}-\frac{X_{n+1}}{D_{n+1}} \\
& =\frac{1}{D_{n} D_{n+1}}\left(X_{n} D_{n+1}-X_{n+1} D_{n}\right) \\
& =\frac{1}{D_{n} D_{n+1}}\left(X_{n}\left(D_{n}+R_{n+1}\right)-\left(X_{n}+\delta_{n+1} R_{X}(n+1)\right) D_{n}\right) \\
& =\frac{1}{D_{n} D_{n+1}}\left(X_{n} R_{n+1}-\delta_{n+1} R_{X}(n+1) D_{n}\right) \\
& =\frac{1}{D_{n} D_{n+1}}\left(X_{n} R_{n+1}-\delta_{n+1} R_{n+1} D_{n}\right) \\
& =\frac{R_{n+1}}{D_{n+1}}\left(Z_{n}-\delta_{n+1}\right) \\
& =Q_{n} \frac{\sum_{i=1}^{n+1} R_{i}}{D_{n+1}}\left(Z_{n}-\delta_{n+1}\right)
\end{aligned}
$$

which yields $\left|\Delta Z_{n}\right| \leq Q_{n}$. Hence, $\mathrm{E}\left(\sup _{k} \sqrt{k}\left|\Delta Z_{k}\right|\right)^{4} \leq \mathrm{E}\left(\sum_{k} k^{2} Q_{k}^{4}\right)<\infty$ by Lemma 5 . Since $\left(\operatorname{Esup}_{k} \sqrt{k}\left|\Delta Z_{k}\right|\right)^{4} \leq \mathrm{E}\left(\sup _{k} \sqrt{k}\left|\Delta Z_{k}\right|\right)^{4}<\infty$, this proves (8a).

Proof of $(8 b)$. We split the proof into four steps.

Step 1. We show that

$\lim _{n \rightarrow \infty} n \sum_{k>n}\left(\Delta M_{k}\right)^{2}=\bar{H} \quad$ almost surely $\quad \Longleftrightarrow \quad \lim _{n \rightarrow \infty} n \sum_{k>n}\left(\Delta Z_{k}\right)^{2}=\bar{H} \quad$ almost surely. 
Lemma 4 shows that $\mathrm{E}\left(\sum_{k>0} \sqrt{k}\left|\Delta A_{k}\right|\right)<\infty$ almost surely; hence,

$$
\mathrm{E}\left(\sum_{k>0} k\left|\Delta A_{k}\right|^{2}\right)<\infty
$$

almost surely and this implies that $\lim _{n \rightarrow \infty} \sum_{k>n} k\left|\Delta A_{k}\right|^{2}=0$ on a set of probability 1 . However, $n\left|\Delta A_{k}\right|^{2} \leq k\left|\Delta A_{k}\right|^{2}$ for $k>n=1,2, \ldots$, and, thus,

$$
\lim _{n \rightarrow \infty} n \sum_{k>n}\left|\Delta A_{k}\right|^{2}=0 \quad \text { almost surely. }
$$

For $n=1,2, \ldots$,

$$
\sum_{k \geq 0} k^{2} Q_{k}^{4} \geq\left(\sqrt{n}\left(\sup _{k>n} Q_{k}\right)\right)^{4}
$$

and, thus, Lemma 5 implies that $\mathrm{P}\left(\sup _{n} \sqrt{n}\left(\sup _{k>n} Q_{k}\right)=\infty\right)=0$, which in turn implies, through (9), that $\mathrm{P}\left(\sup _{n} \sqrt{n}\left(\sup _{k>n} \Delta Z_{k}\right)=\infty\right)=0$. Hence,

$$
\lim _{n \rightarrow \infty}\left|n \sum_{k>n} \Delta Z_{k} \Delta A_{k}\right| \leq\left|\sup _{n} \sqrt{n} \sup _{k>n} \Delta Z_{k}\right| \lim _{n \rightarrow \infty} \sqrt{n} \sum_{k>n}\left|\Delta A_{k}\right|=0
$$

almost surely, where the last equality follows, once again, from Lemma 4 . Since, for $n=$ $1,2, \ldots$,

$$
\left(\Delta M_{n}\right)^{2}=\left(\Delta Z_{n}-\Delta A_{n}\right)^{2}=\left(\Delta Z_{n}\right)^{2}+\left(\Delta A_{n}\right)^{2}-2 \Delta Z_{n} \Delta A_{n},
$$

(10) and (11) imply that

$$
\lim _{n \rightarrow \infty} n \sum_{k>n}\left(\left(\Delta M_{n}\right)^{2}-\left(\Delta Z_{n}\right)^{2}\right)=\lim _{n \rightarrow \infty} n \sum_{k>n}\left(\left(\Delta A_{n}\right)^{2}-2 \Delta Z_{n} \Delta A_{n}\right)=0
$$

on a set of probability 1 . This concludes the proof of the first step.

For the next three steps, we follow the arguments in [3, Theorem 1.1] armed with the results provided by Proposition 1 and Lemma 5.

Step 2. We show that

$$
\begin{gathered}
\lim _{n \rightarrow \infty} n \sum_{k>n}\left(\Delta Z_{k}\right)^{2}=\bar{H} \quad \text { almost surely } \\
\Longleftrightarrow \quad \lim _{n \rightarrow \infty} n \sum_{k>n}\left(Z_{k}-\delta_{k+1}\right)^{2} Q_{k}^{2}=\bar{H} \quad \text { almost surely. }
\end{gathered}
$$

Lemma 7 and (12) imply the almost-sure convergence of $\sum_{n}\left(\Delta Z_{n}\right)^{2}$. Thus, from (9) and Lemma 6, we obtain

$$
\sum_{k>n}\left(\Delta Z_{k+1}\right)^{2}=\sum_{k>n}\left(Z_{k}-\delta_{k+1}\right)^{2} \frac{R_{k+1}^{2}}{D_{k+1}^{2}} \sim \sum_{k>n}\left(Z_{k}-\delta_{k+1}\right)^{2} Q_{k}^{2}
$$

as $n$ grows to $\infty$; this completes the proof of the second step.

Step 3. We show that the almost-sure convergence of

$$
\sum_{k=0}^{m} k\left(\delta_{k+1}-Z_{k}\right)\left(1-Z_{k}\right)^{2}\left(Q_{k}^{X}\right)^{2}
$$


and

$$
\sum_{k=0}^{m} k\left(\delta_{k+1}-Z_{k}\right) Z_{k}^{2}\left(Q_{k}^{Y}\right)^{2}
$$

as $m$ grows to $\infty$ implies that $\lim _{n \rightarrow \infty} n \sum_{k>n}\left(Z_{k}-\delta_{k+1}\right)^{2} Q_{k}^{2}=\bar{H}$ almost surely.

Because of Abel's theorem, the almost-sure convergence of the series (13) and (14) implies that

$$
\begin{gathered}
\lim _{n \rightarrow \infty} n \sum_{k>n}\left(\delta_{k+1}-Z_{k}\right)\left(1-Z_{k}\right)^{2}\left(Q_{k}^{X}\right)^{2}=0, \\
\lim _{n \rightarrow \infty} n \sum_{k>n}\left(\delta_{k+1}-Z_{k}\right) Z_{k}^{2}\left(Q_{k}^{Y}\right)^{2}=0,
\end{gathered}
$$

on a set of probability 1 . Now, from Proposition 1 and the almost-sure convergence of the sequence $\left\{Z_{n}\right\}$ to $Z_{\infty}$, we obtain

$$
\begin{gathered}
\lim _{n \rightarrow \infty} n \sum_{k>n} Z_{k}\left(1-Z_{k}\right)^{2}\left(Q_{k}^{X}\right)^{2}=H_{X} Z_{\infty}\left(1-Z_{\infty}\right)^{2}, \\
\lim _{n \rightarrow \infty} n \sum_{k>n}\left(1-Z_{k}\right) Z_{k}^{2}\left(Q_{k}^{Y}\right)^{2}=H_{Y}\left(1-Z_{\infty}\right) Z_{\infty}^{2},
\end{gathered}
$$

on a set of probability 1 . Equations (15)-(16) yield

$$
\begin{aligned}
\lim _{n \rightarrow \infty} n \sum_{k>n} \delta_{k+1}\left(1-Z_{k}\right)^{2}\left(Q_{k}^{X}\right)^{2} & =H_{X} Z_{\infty}\left(1-Z_{\infty}\right)^{2}, \\
\lim _{n \rightarrow \infty} n \sum_{k>n}\left(1-\delta_{k+1}\right) Z_{k}^{2}\left(Q_{k}^{Y}\right)^{2} & =H_{Y}\left(1-Z_{\infty}\right) Z_{\infty}^{2},
\end{aligned}
$$

almost surely. Since, for all $k \geq 0, Q_{k}=\delta_{k+1} Q_{k}^{X}+\left(1-\delta_{k+1}\right) Q_{k}^{Y}$ and $\delta_{k+1}\left(1-\delta_{k+1}\right)=0$, we have

$$
\begin{aligned}
\lim _{n \rightarrow \infty} & n \sum_{k>n}\left(Z_{k}-\delta_{k+1}\right)^{2} Q_{k}^{2} \\
& =\lim _{n \rightarrow \infty} n \sum_{k>n}\left(\delta_{k+1}\left(1-Z_{k}^{2}\right)\left(Q_{k}^{X}\right)^{2}+\left(1-\delta_{k+1}\right) Z_{k}^{2}\left(Q_{k}^{Y}\right)^{2}\right) \\
& =H_{X} Z_{\infty}\left(1-Z_{\infty}\right)^{2}+H_{Y}\left(1-Z_{\infty}\right) Z_{\infty}^{2} \\
& =\bar{H}
\end{aligned}
$$

on a set of probability 1 .

Step 4 . We prove the almost-sure convergence of the series

$$
\sum_{k=0}^{\infty} k\left(\delta_{k+1}-Z_{k}\right)\left(1-Z_{k}\right)^{2}\left(Q_{k}^{X}\right)^{2}
$$

the proof of the almost-sure convergence of $\sum_{k=0}^{\infty} k\left(\delta_{k+1}-Z_{k}\right) Z_{k}^{2}\left(Q_{k}^{Y}\right)^{2}$ is similar.

For $n=0,1,2, \ldots, R_{X}(n+1)$ is independent of $\sigma\left(\delta_{n+1}, \mathcal{A}_{n}\right)$ and, thus,

$$
\mathrm{E}\left(n\left(\delta_{n+1}-Z_{n}\right)\left(1-Z_{n}\right)^{2} \frac{R_{X}^{2}(n+1)}{\left(D_{n}-D_{0}\right)^{2}} \mid \mathcal{A}_{n}\right)=0 .
$$


Hence,

$$
\begin{aligned}
\mid \mathrm{E}(n & \left.\left(\delta_{n+1}-Z_{n}\right)\left(1-Z_{n}\right)^{2}\left(Q_{n}^{X}\right)^{2} \mid \mathcal{A}_{n}\right) \mid \\
& =\left|\mathrm{E}\left(n\left(\delta_{n+1}-Z_{n}\right)\left(1-Z_{n}\right)^{2}\left(\left(Q_{n}^{X}\right)^{2}-\frac{R_{X}^{2}(n+1)}{\left(D_{n}-D_{0}\right)^{2}}\right) \mid \mathcal{A}_{n}\right)\right| \\
& \leq \mathrm{E}\left(n\left|\delta_{n+1}-Z_{n}\right|\left(1-Z_{n}\right)^{2} R_{X}^{2}(n+1)\left(\frac{1}{\left(D_{n}-D_{0}\right)^{2}}-\frac{1}{\left(D_{n+1}-D_{0}\right)^{2}}\right) \mid \mathcal{A}_{n}\right) \\
& \leq n \beta^{2} \mathrm{E}\left(\frac{1}{\left(D_{n}-D_{0}\right)^{2}}-\frac{1}{\left(D_{n+1}-D_{0}\right)^{2}} \mid \mathcal{A}_{n}\right) \\
& \leq 2 n \beta^{3} \frac{1}{\left(D_{n}-D_{0}\right)^{3}} ;
\end{aligned}
$$

the last inequality holds because

$$
\begin{aligned}
\left(\frac{1}{D_{n}-D_{0}}\right)^{2}-\left(\frac{1}{D_{n+1}-D_{0}}\right)^{2} & =\frac{\left(D_{n+1}-D_{0}\right)^{2}-\left(D_{n}-D_{0}\right)^{2}}{\left(D_{n}-D_{0}\right)^{2}\left(D_{n+1}-D_{0}\right)^{2}} \\
& \leq \frac{2\left(D_{n+1}-D_{0}\right) R_{n+1}}{\left(D_{n}-D_{0}\right)^{2}\left(D_{n+1}-D_{0}\right)^{2}} \\
& \leq \frac{2 \beta}{\left(D_{n}-D_{0}\right)^{3}} .
\end{aligned}
$$

However, $\lim _{n \rightarrow \infty} D_{n} / n=m$ almost surely, as proved in [9, Lemma A.1(iii)]; thus,

$$
\sum_{n} n \mathrm{E}\left(\left(\delta_{n+1}-Z_{n}\right)\left(1-Z_{n}\right)^{2}\left(Q_{n}^{X}\right)^{2} \mid \mathcal{A}_{n}\right)<\infty
$$

on a set of probability 1 .

Next note that, as in [3, Equation (16)],

$$
\mathrm{E}\left(\sum_{n} n^{2} \mathrm{E}\left(\left(\delta_{n+1}-Z_{n}\right)^{2}\left(1-Z_{n}\right)^{4}\left(Q_{n}^{X}\right)^{4} \mid \mathcal{A}_{n}\right)\right) \leq \mathrm{E}\left(\sum_{n} n^{2}\left(Q_{n}^{X}\right)^{4}\right)<\infty
$$

because of Lemma 5. Therefore, Lemma 7 implies that series (13) converges on a set of probability 1 ; this concludes the proof of the fourth step and that of the theorem.

Proof of Theorem 2. Recall that if $\pi$ and $\pi^{\prime}$ are probability distributions on $\mathbb{R}$, the discrepancy metric $d_{D}$ between $\pi^{\prime}$ and $\pi$ is defined as

$$
d_{D}\left(\pi^{\prime}, \pi\right)=\sup _{\text {closed balls } B}\left|\pi^{\prime}(B)-\pi(B)\right| ;
$$

this metric metrizes the weak convergence of a sequence of probability distributions $\left\{\pi_{n}\right\}$ to $\pi$, when the limiting probability distribution $\pi$ is absolutely continuous with respect to the Lebesgue measure on $\mathbb{R}$ (see, e.g. [6]).

The definition of $Z_{\infty}$, Theorem 3.2 of [9], and Theorem 1 imply the existence of $\Omega^{\prime} \in \mathcal{A}$ such that $\mathrm{P}\left(\Omega^{\prime}\right)=1$, and, for all $\omega \in \Omega^{\prime}$,

$$
\lim _{n \rightarrow \infty} Z_{n}(\omega)=Z_{\infty}(\omega)
$$


and

$$
\lim _{n \rightarrow \infty} d_{D}\left(\left(K_{n}\right)(\omega), \mathcal{N}(0, \bar{H}(\omega))\right)=0
$$

where $\bar{H}(\omega)=H(\omega) Z_{\infty}(\omega)\left(1-Z_{\infty}(\omega)\right)$.

By way of contradiction, assume that there is a $p \in(0,1)$ such that $\mathrm{P}\left(Z_{\infty}=p\right)>0$ (for $p \in\{0,1\}$, see [9, Theorem 3.2]). Since

$$
\lim _{n \rightarrow \infty} \mathrm{P}\left(Z_{\infty}=p \mid \mathcal{A}_{n}\right)=\mathbf{1}_{\{p\}}\left(Z_{\infty}\right)
$$

almost surely, there is a set $F \in \mathcal{A}, F \subseteq\left\{Z_{\infty}=p\right\} \cap \Omega^{\prime}$, such that $\mathrm{P}(F)>0$ and, for all $\omega \in F$,

$$
\lim _{n \rightarrow \infty} \mathrm{P}\left(Z_{\infty}=p \mid \mathcal{A}_{n}\right)(\omega)=1 .
$$

Fix $\omega \in F$. For $n=1,2, \ldots$, set $x_{n}=\sqrt{n}\left(Z_{n}(\omega)-p\right)$ and consider the closed ball $B_{n}=\left\{x_{n}\right\}$. Then, for $n=1,2, \ldots$,

$$
d_{D}\left(K_{n}(\omega), \mathcal{N}(0, \bar{H}(\omega))\right) \geq\left|K_{n}(\omega)\left(B_{n}\right)-\mathcal{N}(0, \bar{H}(\omega))\left(B_{n}\right)\right|=K_{n}(\omega)\left(B_{n}\right)
$$

however, $\lim _{n \rightarrow \infty} K_{n}(\omega)\left(B_{n}\right)=1$ because of (18), and this contradicts (17b).

Remark 1. The same argument also works to show that the distribution of the limit composition $V$ of the generalized Pólya urns treated in [3] has no point masses, whenever we can prove that, for the considered urn model, $\mathrm{P}(V=0)=\mathrm{P}(V=1)=0$ and the conditions of Theorem 1.1 of [3] are satisfied.

Proof of Theorem 3. Assume that $m_{v}>0$. Otherwise, it is trivial to prove that $\mathrm{P}\left(Z_{\infty}=\right.$ 1) $=1$.

We will work through a coupling argument that considers two randomly reinforced urns with the same initial composition $(x, y)$. Compositions of the first urn are described by the process $\left\{\left(X_{n}, Y_{n}\right)\right\}$ defined in $(1)$; the composition process $\left\{\left(\widetilde{X}_{n}, \widetilde{Y}_{n}\right)\right\}$ of the second urn is defined by

$$
\begin{aligned}
\widetilde{X}_{n+1} & =\widetilde{X}_{n}+R_{X}(n+1) \widetilde{\delta}_{n+1}, \\
\widetilde{Y}_{n+1} & =\widetilde{Y}_{n}+\widetilde{R}_{Y}(n+1)\left(1-\widetilde{\delta}_{n+1}\right),
\end{aligned}
$$

where, for $n=0,1,2, \ldots, \widetilde{R}_{Y}(n+1)=R_{Y}(n+1)+\left(m_{\mu}-m_{v}\right)$ and $\widetilde{\delta}_{n+1}$ is the indicator of the event $\left\{U_{n+1} \leq \widetilde{X}_{n}\left(\tilde{X}_{n}+\widetilde{Y}_{n}\right)^{-1}\right\}$. The two urns are coupled because the random sequences $\left\{U_{n}\right\}$ and $\left\{\left(V_{n}, W_{n}\right)\right\}$ defining their dynamics through (1) and (19) are the same.

Note that $m_{\mu}=\mathrm{E}\left(R_{X}(n+1)\right)=\mathrm{E}\left(\widetilde{R}_{Y}(n+1)\right)$; hence, Theorem 2 implies that the distribution of $\widetilde{Z}_{\infty}$ has no point masses and, in particular,

$$
\mathrm{P}\left(\widetilde{Z}_{\infty}=0\right)=0
$$

By induction on $n$ we show that $\widetilde{X}_{n} \leq X_{n}$ and that $\widetilde{Y}_{n} \geq Y_{n}$. For $n=0$, the claim is obvious because the two urns have the same initial composition. Assume that the claim is true for $n$. Then

$$
Z_{n}-\widetilde{Z}_{n}=\frac{X_{n}}{X_{n}+Y_{n}}-\frac{\widetilde{X}_{n}}{\widetilde{X}_{n}+\widetilde{Y}_{n}}=\frac{X_{n} \widetilde{Y}_{n}-\tilde{X}_{n} Y_{n}}{\left(X_{n}+Y_{n}\right)\left(\widetilde{X}_{n}+\widetilde{Y}_{n}\right)} \geq 0,
$$


which implies that $\delta_{n+1} \geq \widetilde{\delta}_{n+1}$. Hence,

$$
X_{n+1}-\widetilde{X}_{n+1}=\left(X_{n}-\widetilde{X}_{n}\right)+R_{X}(n+1)\left(\delta_{n+1}-\widetilde{\delta}_{n+1}\right) \geq 0
$$

and

$$
\begin{aligned}
\tilde{Y}_{n+1}-Y_{n+1} & =\left(\tilde{Y}_{n}-Y_{n}\right)+R_{Y}(n+1)\left(\delta_{n+1}-\tilde{\delta}_{n+1}\right)+\left(m_{\mu}-m_{v}\right)\left(1-\widetilde{\delta}_{n+1}\right) \\
& \geq 0 .
\end{aligned}
$$

Therefore, (20) holds for all $n$; hence, $\mathrm{P}\left(Z_{\infty}=0\right) \leq \mathrm{P}\left(\widetilde{Z}_{\infty}=0\right)=0$.

It remains to prove that $\mathrm{P}\left(Z_{\infty} \in(0,1)\right)=0$. To obtain this, we can use the same argument as in [13, Theorem 5], once it has been proved that [13, Equation (11) in the proof of Lemma 4] holds without the assumption of boundedness away from 0 for the supports of the reinforcement distributions. Defining $A_{n}^{*}$ as in (2), this is tantamount to showing that

$$
\lim _{n \rightarrow \infty} \sum_{k=1}^{n} A_{k}^{*}=+\infty
$$

on a set of probability 1 . However, when $m_{\mu}>m_{\nu}$, Lemma 2 shows that, for almost every $\omega \in \Omega$, there is an $a>0$ such that $A_{n}^{*}(\omega) \geq a /\left(D_{0}+n \beta\right)$ eventually; hence, (21) is true.

\section{A final remark on absolute continuity}

Having proved that the distribution of the limit proportion $Z_{\infty}$ of an RRU has no point masses, when $\min (x, y)>0$ and the means of the reinforcement distributions $\mu$ and $v$ are the same, the next obvious question concerns its absolute continuity with respect to the Lebesgue measure. Theorem 2 implies that $\mathrm{P}\left(Z_{\infty} \in S\right)=0$ for all countable sets $S$ in $[0,1]$. The next step would be to show that if $S$ is a Lebesgue null set then $\mathrm{P}\left(Z_{\infty} \in S\right)=0$. Unfortunately, the idea developed in the proof of Theorem 2 cannot be further exploited to produce such a result. In any case, if the closed balls $B_{n}$ appearing in the proof are replaced with the 'holes' of a porous set (for the link between $\sigma$-porous sets and measures, see [12] and [20]), it is possible to show that

$$
\mathrm{P}\left(Z_{\infty} \in S\right)=0
$$

for all $\sigma$-porous sets $S$ in $[0,1]$. Unfortunately, this is not enough to prove that the distribution of $Z_{\infty}$ is absolutely continuous; indeed, $T$-measures are singular with respect to the Lebesgue measure, but they attribute 0 -measure to any $\sigma$-porous set (see, e.g. [17] and [19]).

\section{Acknowledgements}

We thank an anonymous referee and Patrizia Berti, Irene Crimaldi, Luca Pratelli, and Pietro Rigo, who identified a few oversights appearing in a previous version of this paper.

\section{References}

[1] Aletti, G., May, C. And Secchi, P. (2007). On the distribution of the limit proportion for a two-color, randomly reinforced urn with equal reinforcement distributions. Adv. Appl. Prob. 39, 690-707.

[2] Beggs, A. W. (2005). On the convergence of reinforcement learning. J. Econom. Theory 122, 1-36.

[3] Crimaldi, I. (2008). Almost sure conditional convergence for a generalized Pólya urn. Preprint, Dipartimento di Matematica, Università di Bologna. Available at: http://almadl.cib.unibo.it/.

[4] Durham, S. D. AND YU, K. F. (1990). Randomized play-the leader rules for sequential sampling from two populations. Prob. Eng. Inf. Sci. 4, 355-367. 
[5] Durham, S. D., Flournoy, N. And Li, W. (1998). A sequential design for maximizing the probability of a favourable response. Canad. J. Statist. 26, 479-495.

[6] GibBs, A. L. AND Su, F. E. (2002). On choosing and bounding probability metrics. Internat. Statist. Rev. 70, 419-435.

[7] Hopkins, E. ANd Posch, M. (2005). Attainability of boundary points under reinforcement learning. Games Econom. Behavior 53, 110-125.

[8] Li, W., Durham, S. D. And Flournoy, N. (1996). Randomized Pólya urn designs. In Proc. Biometric Section Amer. Statist. Assoc., American Statistical Association, Alexandria, VA, pp. 166-170.

[9] May, C. And Flournoy, N. (2008). Asymptotics in response-adaptive designs generated by a two-color, randomly reinforced urn. Ann. Statist. 32, 1058-1078.

[10] May, C., Paganoni, A. and Secchi, P. (2005). On a two color generalized Pólya urn. Metron 63, 115-134.

[11] May, C., Paganoni, A. and Secchi, P. (2007). Response-adaptive designs targeting the best treatment for clinical trials with continuous responses. In S. Co. 2007 5th Conf. - Complex Models and Computational Intensive Methods for Estimation and Prediction, Cluep, Padova, pp. 326-331.

[12] Mera, M. E., Morán, M., Preiss, D. And Zajíček, L. (2003). Porosity, $\sigma$-porosity and measures. Nonlinearity 16, 247-255.

[13] Muliere, P., Paganoni, A. And Secchi, P. (2006). A randomly reinforced urn. J. Statist. Planning Infer. 136, 1853-1874.

[14] Paganoni, A. M. And Secchi, P. (2007). A numerical study for comparing two response-adaptive designs for continuous treatment effects. Statist. Meth. Appl. 16, 321-346.

[15] Pemantle, R. (1990). A time-dependent version of Pólya's urn. J. Theoret. Prob. 3, 627-637.

[16] Pemantle, R. and Volkov, S. (1999). Vertex-reinforced random walk on $\boldsymbol{Z}$ has finite range. Ann. Prob. 27, 1368-1388.

[17] ProkaJ, V. (2001/02). On a construction of J. Tkadlec concerning $\sigma$-porous sets. Real Anal. Exchange 1, 269273.

[18] RaC̆KAUSKas, A. (1990). On probabilities of large deviations for martingales. Litovsk. Mat. Sb. 30, 784-795 (in Russian). English translation: Lithuanian Math. J. 30 (1991), 376-384.

[19] Tkadlec, J. (1986/87). Construction of a finite Borel measure with $\sigma$-porous sets as null sets. Real Anal. Exchange 1, 349-353.

[20] ZAJíčEK, L. (2005). On $\sigma$-porous sets in abstract spaces. Abstr. Appl. Anal. 5, 509-534. 